La Revue

des Droits

de l'Homme

\section{La Revue des droits de l'homme}

Revue du Centre de recherches et d'études sur les droits fondamentaux

Actualités Droits-Libertés | 2010

\title{
La seule appartenance à une organisation « terroriste » ne justifie pas d'une exclusion automatique du statut de réfugié
}

STATUT DE REFUGIE ET TERRORISME (CJUE)

\section{Caroline Lantero}

\section{OpenEdition}

Journals

Édition électronique

URL : http://journals.openedition.org/revdh/3623

DOI : $10.4000 /$ revdh.3623

ISSN : 2264-119X

Éditeur

Centre de recherches et d'études sur les droits fondamentaux

Référence électronique

Caroline Lantero, « La seule appartenance à une organisation « terroriste » ne justifie pas d'une exclusion automatique du statut de réfugié ", La Revue des droits de l'homme [En ligne], Actualités Droits-Libertés, mis en ligne le 28 novembre 2010, consulté le 20 avril 2019. URL : http:// journals.openedition.org/revdh/3623 ; DOI : 10.4000/revdh.3623

Ce document a été généré automatiquement le 20 avril 2019.

Tous droits réservés 


\title{
La seule appartenance à une organisation « terroriste » ne justifie pas d'une exclusion automatique du statut de réfugié
}

\author{
STATUT DE REFUGIE ET TERRORISME (CJUE)
}

\section{Caroline Lantero}

1 Après avoir clarifié les conditions d'octroi de la protection subsidiaire (C-465/07 Elgafaji contre Staatssecretaris van Justitie, arrêt du 17 février 2009) et analysé les conditions de cessation du statut de réfugié pour des raisons liées au changement des circonstances qui ont justifié sa reconnaissance (C-175/08, C-176/08, C-178/08 et C-179/08 Aydin Salahadin Abdulla e.a. contre Allemagne, arrêt du 2 mars 2010), la Cour de justice de l'Union européenne intervient à nouveau pour préciser les modalités d'application de la directive $n^{\circ}$ 2004/83/CE du Conseil du 29 avril 2004 dite directive "qualification», et apporte incidemment un éclairage sur l'interprétation de la Convention de Genève de 1951 relative au statut du réfugié qui est, aux termes même de la directive, $3^{\mathrm{e}}$ considérant, « la pierre angulaire du régime international de protection des réfugiés ».

2 Confrontée à un candidat au statut de réfugié d'une part, et à un réfugié reconnu d'autre part, ayant respectivement appartenu à des organisations citées par la position commune 2001/931/PESC relative à l'application de mesures spécifiques en vue de lutter contre le terrorisme, la cour administrative fédérale allemande a saisi la Cour de justice de cinq questions préjudicielles. La Directive a pour objet d'établir des normes minimales relatives, d'une part, aux conditions que doivent remplir les demandeurs d'asile pour pouvoir bénéficier d'une protection internationale et, d'autre part, au contenu de la protection accordée. Et à l'instar de la Convention de Genève, la Directive prévoit d'exclure une personne du statut de réfugié lorsqu'il y des raisons sérieuses de penser qu'elle a commis un "crime grave de droit commun" ou s'est rendue coupable d'" agissements contraires aux buts et aux principes des Nations unies ".Étaient en jeu la nonreconnaissance du statut pour l'un et la révocation pour l'autre, et il était principalement 
demandé à la Cour de se prononcer sur la possibilité que l'appartenance à une telle liste soit constitutive d'un crime grave de droit commun ou d'un agissement contraire aux buts et principes des Nations Unies au sens de l'article 12, paragraphe 2 de la directive.

Il faut noter à titre liminaire que sur le plan des principes l'inscription des clauses d'exclusion du statut de réfugié, tant dans la Directive "qualification» que dans la Convention de Genève, sont considérées comme donnant tout son sens au concept de l'asile, et le Haut-Commissariat des Nations Unies pour les réfugiés (HCR) résume ainsi ce qui s'est dégagé de la Conférence des plénipotentiaires lors de la rédaction de la Convention dans une note de son comité exécutif: "L'idée selon laquelle une personne ne mérite pas la protection en qualité de réfugié a trait aux liens intrinsèques existant entre les idées d'humanité, d'équité et le concept de réfugié. Les objectifs primordiaux de ces clauses d'exclusion sont de priver de cette protection les auteurs d'actes haineux et de crimes graves de droit commun et de préserver le pays d'accueil de l'entrée de criminels qui présentent un danger pour la sécurité de ce pays. Si la protection fournie par le droit des réfugiés permettait d'offrir la protection aux auteurs de graves délits, la pratique de la protection internationale entrerait directement en conflit avec le droit national et international et s'inscrirait en faux contre la nature humanitaire et pacifique du concept de l'asile. Sous cet angle, les clauses d'exclusion contribuent à sauvegarder l'intégrité du concept de l'asile. » On relèvera également que les questions opposant les enjeux de la lutte contre le terrorisme aux principes gouvernant l'expulsion des étrangers, le retrait ou l'exclusion du statut de réfugié ou de la protection subsidiaire se multiplient (v. Cour EDH, 3e Sect. 20 juillet 2010, A. c. Pays-Bas, Req. $n^{\circ}$ 4900/06 - ADL du 26 juillet 2010 ; Cour EDH, $5^{e}$ Sect. 3 décembre 2009, Daoudi c. France, Req. $n^{\circ}$ 19576/08 ADL du 3 décembre $2009 ; \mathrm{CE}, 8$ octobre 2010,10e et $9^{\mathrm{e}}$ sous-sections réunies, Kamel Daoudi (n - 338505) ADL du 13 octobre 2010).

Sur le plan de la compétence rationae temporis de la Cour, une difficulté était soulevée dès lors que le droit national allemand appliqué au cas d'espèce était antérieur à l'entrée en vigueur de la directive. Mais la Cour a rappelé qu'elle était compétente dans un souci de convergence des interprétations futures, et au regard du fait que la législation allemande alors en vigueur avait inséré des clauses "correspondant en substance à celles» de la Convention de Genève (article $1 \mathrm{~F}$ ), et du fait que les clauses d'exclusion inscrites dans la Directive « correspondent, également, en substance » à celle de la Convention de Genève (par analogie, CJUE, 2 mars 2010, Aydin Salahadin Abdulla e.a. contre Allemagne, précité). Dès lors, de substance en substance, la CJUE est compétente pour interpréter la Convention de Genève et le droit national, ce qui est susceptible de constituer une plus-value pour la première, seulement défendue par la doctrine et le HCR.

Plusieurs éclairages sont ainsi apportés par la Cour de justice dans cet avis.

\section{$1 \%$ Sur les notions de crimes graves de droit commun et d'agissements contraires aux buts et principes des Nations Unies}

6 Aux termes de l'article 12 paragraphe 2 de la directive « qualification » et de l'article $1 \mathrm{~F}$ de la Convention de Genève, le bénéfice de la protection ne s'applique pas - entre autres - à un individu dont il y a «de sérieuses raisons de penser » qu'il a commis un crime grave de droit commun en dehors du pays d'accueil avant d'y être admis, ou qu'il s'est rendu " coupable d'agissements contraires aux buts et principes des Nations Unies ». Si la Directive est 
légèrement plus précise que la Convention sur ces deux qualifications, celles-ci ont pu être source de difficulté.

7 - L'_expression_ " crime grave de droit commun » est généralement entendue comme tout ce qui n'est pas un crime politique. Le HCR a précisé qu'il fallait d'une part «trouver un équilibre entre la nature du crime présumé commis par le demandeur d'asile et le degré de persécution qu'il craint de subir " (HCR, Note d'information sur l'article 1 de la Convention de Genève) et d'autre part, vérifier que l'acte accompli n'est pas «tout à fait hors de proportion avec l'objectif prétendument visé » (paragraphe 152 du Guide des procédures et critères à appliquer pour déterminer le statut de réfugié au regard de la Convention de 1951 et du Protocole de 1967 relatifs au statut des réfugiés), ce qui concerne précisément le terrorisme (HCR, Application des clauses d'exclusion). La notion « d'agissements contraires aux buts et principes des Nations Unies » est un peu hybride en ce qu'elle pourrait se retrouver dans l'ensemble des clauses d'exclusion déjà prévues tant par la Convention que par la Directive (crime contre la paix, crime de guerre, crime grave de droit commun,). L'énoncé de ces buts et principes se trouvant dans la Charte des Nations Unies, on peut à tout le moins supposer que de tels agissements ne peuvent être que le fait d'un agent d'un État membre des Nations Unies, ce qui semble a priori exclure le terrorisme.

8 La Cour de justice confirme en premier lieu que les « actes de nature terroriste (...) même s'ils sont commis dans un objectif prétendument politique, doivent être regardés comme des crimes graves de droit commun » (point 81) et estime en deuxième lieu que, puisque figurent dans les résolutions des Nations Unies des «mesures visant à éliminer le terrorisme », les actes de terrorisme international sont également "d'une manière générale, et indépendamment de la participation d'un État, contraires aux buts et principes des Nations Unies » (point 83). Elle rejette en troisième lieu fermement, en tout état de cause pour les actes de terrorisme, le critère de proportionnalité évoqué plus haut en jugeant que l'autorité compétente qui aboutit à la conclusion qu'un individu doit être exclu du statut sur le fondement de la gravité des actes commis, n'a pas à " procéder à un examen de proportionnalité impliquant de nouveau une appréciation du niveau de gravité des actes commis» (point 109). Elle précise en quatrième lieu que ces actes doivent avoir été commis avant de trouver refuge, et que la circonstance que l'individu continue ou non de représenter un danger pour l'État d'accueil est sans incidence sur l'applicabilité d'une clause d'exclusion. La question d'une menace actuelle pour l'État d'accueil est en effet régie par d'autres dispositions de la directive et de la Convention (points 100-105).

\section{$2 \%$ Sur la portée des « sérieuses raisons de penser que $»$}

9 Mais la Cour précise surtout la portée des "sérieuses raisons de penser que " l'individu se soit livré à de tels actes disqualifiants, et ne donne à la «black list» européenne des organisations terroristes qu'une valeur d'indice. "La seule circonstance que la personne concernée a appartenu à une telle organisation ne saurait avoir comme conséquence automatique qu'elle doive être exclue du statut du réfugié» (point 88). Il faut cependant relever que la Cour de justice appuie cette position sur le fait que la position commune ayant établi ladite liste a été adoptée "en dehors du cadre que la directive a instauré dans le respect de la convention de Genève » (point 89). 


\section{$3 \%$-Sur l'examen de la demande de statut de réfugié}

10 A la suite du HCR qui a toujours insisté pour que les clauses d'inclusion de la Convention de Genève soient chronologiquement examinées avant les clauses d'exclusion, la Cour de Justice tire les mêmes enseignements des dispositions de la directive et estime que les clauses d'exclusions porte sur des faits « commis par l'intéressé, qui remplit par ailleurs les critères pour obtenir le statut de réfugié » (point 87), ce qui sous-tend l'obligation d'étudier l'éligibilité au statut en premier lieu. La Cour ajoute logiquement que l'examen des clauses d'exclusion suppose une "évaluation individuelle de faits précis » (point 91), laquelle est par définition peu compatible avec les conditions dans lesquelles peuvent être dressées des listes d'organisations terroristes. Ainsi l'appartenance à une telle liste n'est qu'un élément de preuve qui doit s'accompagner d'un examen individuel portant notamment sur le rôle effectivement joué par la personne au sein de l'organisation, sa position effectivement tenue, son degré de connaissance des activités de l'organisation, et les éventuelles pressions auxquelles elle aurait pu être soumise.

\section{4\%- Sur le droit souverain des États à accorder l'asile}

11 La Cour de justice précise enfin la portée du droit souverain des États à accorder l'asile à des individus qui en sont pourtant «indignes» aux termes des clauses d'exclusion de la Directive «qualification" et de la Convention de Genève. Selon la Cour, cette clause de souveraineté, telle qu'inscrite à l'article 3 de la directive n'est applicable que pour autant qu'elle n'entre pas en contradiction avec l'ensemble des normes de la directive. Fermant la boucle sur le concept de crédibilité et d'intégrité de l'institution asile, la Cour admet la possibilité de reconnaitre souverainement un asile constitutionnel aux individus, « ̀̀ titre discrétionnaire et par bienveillance ou pour des raisons humanitaires » (point 118), dans la seule mesure où une telle protection ne se confond pas avec le statut de réfugié au sens de la Directive (point 119).

CJUE, 9 novembre 2010, affaires jointes C-57/09 et C-101/09, Allemagne / B et Allemagne / D

\section{AUTEUR}

\section{CAROLINE LANTERO}

Maître de conférences à l'Université de Clermont-Ferrand 